\title{
Congenital nephrotic syndrome in a Hispanic Guatemalan newborn associated with a NPHS1 variant: A case report
}

\author{
THU T. TRAN, VIJAY G. LINGA, MOHAMMED A.I. AL-OBAIDE, DANIELLA BELLO-GERMINO, \\ MEHAR HODA, OLUBUKUNOLA ADESANYA and TETYANA L. VASYLYEVA \\ Department of Pediatrics, Texas Tech University Health Sciences Center, Amarillo, TX 79106, USA
}

Received September 7, 2021; Accepted November 5, 2021

DOI: 10.3892/br.2021.1487

\begin{abstract}
Congenital nephrotic syndrome (CNS) is an autosomal recessive disorder usually detected in the first 3 months of life when the syndromes effects manifest, including edema and a failure to gain weight. A baby boy was admitted to the Neonatal Intensive Care Unit for prematurity (35 weeks) with unremarkable maternal prenatal laboratory tests. The patient had persistent systemic hypertension, hypoproteinemia, hypoalbuminemia and nephrotic range proteinuria. CNS was diagnosed, and genetic testing showed a homozygous variant, c.3024A $>$ G (AGA $>$ AGG) in exon 22 of the nephrin locus. Bioinformatics analysis suggested the genetic condition was likely a result of malfunctional DNA binding sites of transcription factors FOXL1 and FOXC1.
\end{abstract}

\section{Introduction}

Congenital nephrotic syndrome (CNS) is a rare autosomal recessive disorder. The most common type of CNS is the Finnish type, a disorder characterized by massive proteinuria detected at birth, a large placenta, marked edema and radial dilatation of the proximal tubules (1). The incidence of CNS in Finland is estimated to be 1 in 8,200 live births, and is considered to be lower in other countries. A high incidence of CNS has also been reported in certain regions of the United States, for example, among the Old Order Mennonites in Lancaster, PA (2). Contributing mutations to CNS mostly occur in a panel of five genes: nephrin (NPHS1), podocin (NPHS2), Wilms tumor (WT1), laminin (LAMB2) and phospholipase C epsilon 1 (PLCE1) (3). Previous research has tended to focus on mutations in NPHS1, as it is the main gene involved in CNS of the Finnish type, in an autosomal recessive manner $(3,4)$. However, other studies showed that whilst NPHSI gene mutations can cause CNS in non-Finnish individuals, they are a less common cause than NPHS2,WT1,LAMB2 and PLCE1

Correspondence to: Professor Tetyana L. Vasylyeva, Department of Pediatrics, Texas Tech University Health Sciences Center, 1400 S Coulter Street, Amarillo, TX 79106, USA

E-mail: tetyana.vasylyeva@ttuhsc.edu

Key words: congenital nephrotic syndrome, newborn, NHPS1, variant, regulatory sequences mutations (3). Previous studies have reported NPHS1 mutational analysis suggests that abnormal or inefficient signaling through the nephrin-podocin complex contributes to podocyte dysfunction and proteinuria (5). Typically, the histological lesions of CNS are detected after 3 months of age when symptoms appear, such as failure to gain weight and edema (1). In this case report, the case of a newborn with an early diagnosis of Finnish variant CNS, born to a Guatemalan mother with a NPHS1 variant, is described, and was determined to be most likely the result of malfunctional DNA binding sites for transcription factors in the NPHS1 locus.

\section{Case report}

A baby boy was delivered vaginally at 35.1 weeks gestational age, based on a 24.1-week ultrasound, to a 22-year-old gravida mother from Guatemala. The mother had previously delivered a child prematurely at 32 weeks via cesarean section who died in a Guatemalan hospital for unknown reasons within the first month of life. Her prenatal laboratory tests were unremarkable, and she denies any illicit drug use or alcohol/tobacco use. The mother had late, limited prenatal care that began at 24 weeks, with a limited number of visits. The pregnancy was complicated by membrane rupture $25 \mathrm{~h}$ before delivery. Birth weight was $2.030 \mathrm{~kg}$ (12th percentile average size for gestational age), and suture separation was evident. He was admitted to the Neonatal Intensive Care Unit (NICU) for prematurity.

On day of life (DOL) 2, the infant's creatinine was elevated $(1.30 \mathrm{mg} / \mathrm{dl})$ and hypoproteinemia $(3.1 \mathrm{~g} / \mathrm{dl})$ and hypoalbuminemia $(1.2 \mathrm{~g} / \mathrm{dl})$ were evident. Findings were attributed to poor maternal nutrition and expressed breast milk was fortified with NeoSure ${ }^{\circledR}$ to $27 \mathrm{kcal} /$ ounce. Hypoalbuminemia and hypoproteinemia persisted. Bicitra ( $3 \mathrm{mEq} / \mathrm{kg} /$ day) administration was initiated on DOL 9 to correct metabolic acidosis due to a bicarbonate level of $15 \mathrm{mEq} / 1$. On DOL 16, urinalysis revealed $3+$ protein and $3+$ glucose. The next day a urine protein to creatinine ratio of 59.23 was indicative of nephrotic syndrome.

Transthoracic echocardiogram on DOL 9 showed a patent foramen ovale with a left to right shunt and mild left ventricular hypertrophy. On further workup for hypertension, renin and aldosterone levels were found to be elevated at $130 \mathrm{ng} / \mathrm{dl}$ and $428 \mathrm{ng} / \mathrm{dl}$, respectively. Due to persistent hypertension (since DOL 1) above the 95 th percentile, captopril $(0.05 \mathrm{mg} / \mathrm{kg} / 8 \mathrm{~h})$ was administered beginning on DOL 16 . The renal ultrasound 
was unremarkable. His urinalysis revealed normal $\mathrm{pH}$, which was inadequate in the setting of acidosis.

At 3 months of life, thyroid hormone levels and antithrombin III activity levels started to decrease, which is typical for this condition (6). The patient was placed on levothyroxine, and was treated with albumin infusions, angiotensin-converting enzyme inhibitors (captopril), and nonsteroidal anti-inflammatory drug (Indomethacin) with increased dietary protein supplementation starting on DOL 22. His serum immunoglobulin levels ( $\operatorname{IgG}, \operatorname{IgA}, \operatorname{Ig} M$ and $\operatorname{IgE}$ ) were low and required IVIG treatment through a port-a-cath. He was placed on oral supplementation for copper and iron. Mild periorbital and scrotal edema were first observed on DOL 36. With the identification of the early onset of nephrotic syndrome, genetic testing was performed to assess possible NPHS1, NPHS2, WT1, LAMB2 and PLCE1 mutations.

The patient's genomic DNA was analyzed by Athena Diagnostics via PCR amplification of purified genomic DNA, followed by Sanger DNA sequencing of the gene's coding region. In addition, at least 10 bases of intronic DNA on either side of each exon containing the highly conserved exon-intron splice junctions were also sequenced. No variants were detected in NPHS2, WT1, LAMB2 and PLCE1 (Table I). A positive homozygous variant was detected in NPHS1, mRNA (NCBI Reference Sequence: NM_004646.3) located at c.3024A $>$ G (Fig. S1). Up-to-date analysis by the variant scientists of Athena Diagnostics showed NPHS1 c.3024A $>\mathrm{G}$ is a synonymous variant of uncertain significance, as the available data (Score, 4; range 1-7, benign-pathogenic, respectively) was insufficient to determine the clinical significance of the variant at this time. ClinVar-NCBI showed two cases of the same c.3024A $>\mathrm{G}$ type with uncertain significance (Table II); one is an entry of the reported variant in the present study (variation ID: 429811). Up-to-date searches for the criteria provided in ClinVar for variation ID: 429811, NM_004646.3 (NPHS1):c.3024A>G (p.Arg1008=) showed multiple submissions, the most recent of which was Sep 26th, 2021. The substitution of $A>G$ occurs in the AGA codon for arginine; allele $\mathrm{A}$, mapped in the third position of the triplet at position 3,180 in the NPHS1 mRNA (NM_004646_3) generating a degenerate redundant AGG codon (Fig. 1A). c.3024 indicates the allele's position in the coding sequence (Fig. 1B). The variant was identified as a homozygous variant within NPHS1 exon 22 (Fig. 1C). There can be little doubt that the CNS case was associated with this NPHSI variant. Thus, it was hypothesized that the generated mutation may cause CNS by disturbing the expression of NPHS1, via alteration of the regulatory sequences, which is a likely explanation for the genetic condition. The motifs of the transcriptional factors binding sites (TFBSs) in NPHS1 exon-22 and adjacent introns' sequences were analyzed using JASPAR $2020(7,8)$. The analysis revealed TFBSs presence for at least 10 types of binding motifs specific to transcription factors in exon 22 and the neighboring introns' sequences (Fig. 1C). The c.3024A $>\mathrm{G}$ variant was mapped at TFBSs for FOXL1 and FOXC1. A further point is the c.3024A $>\mathrm{G}$ mutation is close to mammalian-wide interspersed repeats (MIRs), 46 bases away from NPHS1 exon 22 (Fig. S2), the data has been adapted from Genome Browser UCSC (genome.ucsc.edu/). The main function of MIRs is exonization, the generation of new exons from intronic DNA sequences.
Additionally, MIRs are considered as major donors of TFBSs $(9,10)$. Athena Diagnostics computational tools yielded predictions that this homozygous variant may result in the gain of a cryptic splice site without affecting the natural splice sites. Intriguingly, the homozygous variant increased the double helix stability (Fig. S3) as measured by oligo calculator in the atdbio website (atdbio.com/tools/oligo-calculator) in terms of the standard free energy change; such changes are associated with diseases (11). It is hypothesized that the child did not have a de novo mutation, as the family lost their first child, who presented with a very similar clinical condition. The sequence validation was not obtainable for the parents, but was likely to be heterozygous for the NPHS1 variant.

\section{Discussion}

The present CNS case highlights a rare disease with an indicator of suspicion regarding the genetic cause of the disease. Sanger sequencing is an established method and is used for identification and validation of the presence of the homozygous mutation; it is unlikely the mutation is de novo, but is instead from heterozygous parents. Sanger sequencing is used to identify the pathogenic variation in various diseases $(12,13)$. Although the patient showed classic symptoms of CNS $(1,14)$, the detected homozygous c.3024A $>\mathrm{G}$ variant produces a degenerate codon with a genetic condition of uncertain significance. However, keeping in mind the clinical manifestations associated with CNS and the fact that no mutations were found in the other four tested genes associated with CNS (NPHS2, WT1, LAMB2 and $P L C E 1)(3,14)$, the potential involvement of regulatory sequences in NPHS1 exon 22 at the site of mutation is thus discussed further.

The bioinformatics analysis showed that the $A>G$ change caused by c.3024A $>\mathrm{G}$ mutation could alter transcription binding sites; specifically, the composition of two motifs specific to transcription factors FOXL1 and FOXC1. The deregulation of FOX transcription factors leads to congenital disorders $(15,16)$, and consequently, these changes may cause potential malfunction of the transcription process in NPHS1 exon 22.

Intriguingly, previous studies have shown that exons have regulatory activity in addition to their coding activity. As early as 1997, the DNase I hypersensitive sites specific DNA sequences related to the transcriptional activity were identified in coding exons in mice (17). More recently, exons have been shown to mediate activation of transcription starts (18) and have TFBS sequences (19-21). Not surprisingly, recent versions of genomics websites show regulatory sequences spanning coding sequences, for example NPHS1 ENSG00000161270 and several other genes. Furthermore, a recent study found that a transcription factor's binding affinity in exons is weak, but improves in the noncoding sequences of DNA (22). It is quite possible that genetic variants may affect exonic splicing regulatory sequences and consequently disrupt pre-mRNA splicing and initiate genetic diseases $(23,24)$.

To conclude, a rare NPHS1 gene variant (25) is described, which likely caused a disruption in regulatory sequences, TFBSs and cryptic splice sites $(15-17,24,26,27)$ associated with $\mathrm{CNS}$. The c.3024A $>\mathrm{G}$ variant modified the two transcription factors', FOXL1 and FOXC1, binding sites in the NPHS1 exon 22 sequence, and may have influenced MIRs functions $(9,10)$. Consequently, the mutation is likely the cause of dysregulated NPHS1 expression. The reported case should increase awareness 
Table I. Panel of the 5 genes tested for variants by DNA sequencing in the congenital nephrotic syndrome case study. Only one variant was identified in the nephrin gene, NPHS1.

\begin{tabular}{|c|c|c|c|c|c|}
\hline Gene name: Description & $\begin{array}{c}\text { NCBI } \\
\text { gene ID }\end{array}$ & $\begin{array}{c}\text { NCBI } \\
\text { nucleotide ID }\end{array}$ & OMIM no. & Variant & $\begin{array}{c}\text { Clinical } \\
\text { significance }\end{array}$ \\
\hline$L A M B 2$ : Laminin subunit $\beta 2$ & 3913 & NM_002292_3 & OMIM 150325 & No variant detected & - \\
\hline $\begin{array}{l}\text { NPHS1: NPHS1 adhesion } \\
\text { molecule, nephrin }\end{array}$ & 4868 & NM_004646_3 & OMIM 802716 & c. $3024 \mathrm{~A}>\mathrm{G}$ & $\begin{array}{l}\text { Variant of uncertain } \\
\text { significance }\end{array}$ \\
\hline $\begin{array}{l}\text { NPHS2: Stomatin family } \\
\text { member, podocin }\end{array}$ & 7827 & NM_014626_3 & OMIM 804768 & No variant detected & - \\
\hline PLC1: Phospholipase $\mathrm{C} \gamma 1$ & 5335 & NM_018341_3 & OMIM 808414 & No variant detected & - \\
\hline WT1: Transcription factor & 7490 & NM_024428_2 & OMIM 807102 & No variant detected & - \\
\hline
\end{tabular}

Table II. Criteria and submitters of NPHS1 c.3024 A>G variant in ClinVar. Data adapted from ClinVar-NCBI.

Variant location in coding sequence of NPHS1 mRNA NM_004646.3
Collection method
Submitter, submission date
Clinical

significance
Submission accession no.

\section{c. $3024 \mathrm{~A}>\mathrm{G}$}

GRCh37: Chr19:36330224

Clinical testing

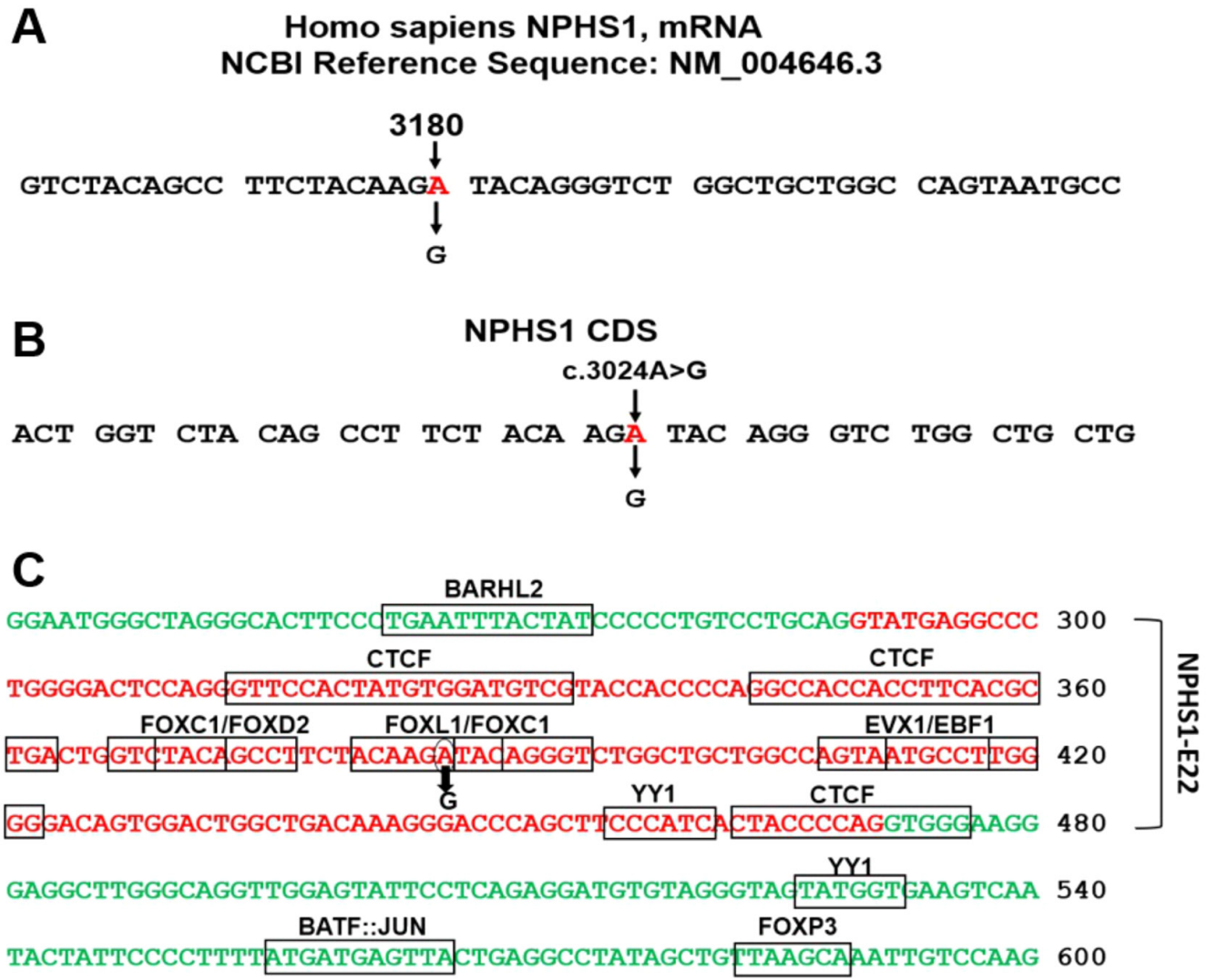

Figure 1. Molecular characterization of the NPHS1 c.3024A>G variant. (A) Location of the missense mutation in the NPHS1 mRNA at position 3,180. (B) Location of the variant in the NPHS1 coding sequence at position 3,024. (C) Bioinformatics analysis of NPHS1-E22 shown in red letters. The green letters refer to the adjacent introns' sequences. The identified sequences of TFBSs are marked. The encircled base 'A' represents the wild type allele whereas 'G' is the mutated allele. JASPAR 2020 was used for identification of TFBSs in the NPHS1-E22 sequence. NPHS1, nephrin; NPHS1-E22, NPHS1 Exon 22; TFBS, transcriptional factors binding site. 
of the early diagnosis of CNS in non-Finish populations. The study may encourage further work to investigate the clinical significance of the c.3024A $>\mathrm{G}$ variant and potential involvement of exonic splicing regulatory sequences and other regulatory sequences in genetic conditions of uncertain significance.

\section{Acknowledgements}

The authors greatly appreciate the variant's information provided by Miss Meagan Nashawaty, MS, Certified Genetic Counselor (CGC), Athena Diagnostics, Marlborough, MA, USA.

\section{Funding}

No funding was received.

\section{Availability of data and materials}

The datasets used and/or analyzed during the current study are available from the corresponding author on reasonable request, and the ClinVar-NCBI repository [ncbi.nlm.nih. gov/clinvar/variation/429811/].

\section{Authors' contributions}

TLV analyzed and interpreted the clinical patient data. MAIAO was responsible for TFBSs, MIRs and homozygous variant double helix stability analyses. TTT, VGL, DBG, MH and OA cared for the patient and assisted in writing the case report. TLV and MAIAO wrote and revised the manuscript. All authors have read and approved the final manuscript. TLV and MAIAO confirm the authenticity of all the raw data.

\section{Ethics approval and consent to participate}

Texas Tech University Health Sciences Center does not require ethical approval for reporting individual cases or case studies.

\section{Patient consent for publication}

Written informed consent was obtained from the patient' parents for publication of anonymized data.

\section{Competing interests}

The authors declare that they have no competing interests.

\section{References}

1. Jalanko H: Congenital nephrotic syndrome. Pediatr Nephrol 24: 2121-2128, 2009.

2. Bolk S, PuffenbergerEG, Hudson J, Morton DH and Chakravarti A: Elevated frequency and allelic heterogeneity of congenital nephrotic syndrome, Finnish type, in the old order Mennonites. Am J Hum Genet 65: 1785-1790, 1999.

3. Preston R, Stuart HM and Lennon R: Genetic testing in steroid-resistant nephrotic syndrome: Why, who, when and how? Pediatr Nephrol 34: 195-210, 2019.

4. Fogo AB, Lusco MA, Najafian B and Alpers CE: AJKD Atlas of Renal Pathology: Congenital nephrotic syndrome of finnish type. Am J Kidney Dis 66: e11-e12, 2015.

5. Gigante M,Piemontese M, Gesualdo L, Iolascon A and Aucella F: Molecular and genetic basis of inherited nephrotic syndrome. Int J Nephrol 2011: 792195, 2011.
6. Golob V, Nosan G, Bertok S, Frelih M, Boštjanči E and Rus R: A novel mutation of congenital nephrotic syndrome in a Slovenian child eventually receiving a renal transplant. Croat Med J 62: 187-191, 2021.

7. KhanA,Fornes O,Stigliani A,GheorgheM,Castro-MondragonJA, van der Lee R, Bessy A, Chèneby $\mathrm{J}$, Kulkarni SR, Tan G, et al: JASPAR 2018: Update of the open-access database of transcription factor binding profiles and its web framework. Nucleic Acids Res 46: D260-D266, 2018.

8. Fornes O, Castro-Mondragon JA, Khan A, van der Lee R, Zhang X, Richmond PA, Modi BP, Correard S, Gheorghe M, Baranašić D, et al: JASPAR 2020: Update of the open-access database of transcription factor binding profiles. Nucleic Acids Res 48: D87-D92, 2020.

9. Krull M, Petrusma M, Makalowski W, Brosius J and Schmitz J: Functional persistence of exonized mammalian-wide interspersed repeat elements (MIRs). Genome Res 17: 1139-1145, 2007.

10. Jjingo D, Conley AB, Wang J, Mariño-Ramírez L, Lunyak VV and Jordan IK: Mammalian-wide interspersed repeat (MIR)-derived enhancers and the regulation of human gene expression. Mob DNA 5: 14, 2014

11. Khan MT, Ali S, Zeb MT, Kaushik AC, Malik SI and Wei DQ: Gibbs free energy calculation of mutation in pnca and rpsa associated with pyrazinamide resistance. Front Mol Biosci 7: 52, 2020

12. Al Qahtani NH, AbdulAzeez S, Almandil NB, Fahad Alhur N, Alsuwat HS, Al Taifi HA, Al-Ghamdi AA, Rabindran Jermy B, Abouelhoda M, Subhani S, et al: Whole-genome sequencing reveals exonic variation of ASIC5 gene results in recurrent pregnancy loss. Front Med (Lausanne) 8: 699672, 2021.

13. Majidi S, Fouts A, Pyle L, Chambers C, Armstrong T, Wang Z, Batish SD, Klingensmith G and Steck AK: Can biomarkers help target maturity-onset diabetes of the young genetic testing in antibody-negative diabetes? Diabetes Technol Ther 20: 106-112, 2018.

14. Caridi G, Gigante M, Ravani P, Trivelli A, Barbano G, Scolari F, Dagnino M, Murer L, Murtas C, Edefonti A, et al: Clinical features and long-term outcome of nephrotic syndrome associated with heterozygous NPHS1 and NPHS2 mutations. Clin J Am Soc Nephrol 4: 1065-1072, 2009.

15. Golson ML and Kaestner KH: Fox transcription factors: From development to disease. Development 143: 4558-4570, 2016.

16. Katoh M and Katoh M: Human FOX gene family (Review). Int J Oncol 25: 1495-1500, 2004

17. Neznanov N, Umezawa A and Oshima RG: A regulatory element within a coding exon modulates keratin 18 gene expression in transgenic mice. J Biol Chem 272: 27549-27557, 1997.

18. Fiszbein A, Krick KS, Begg BE and Burge CB: Exon-mediated activation of transcription starts. Cell 179: 1551-1565.e17, 2019.

19. Stergachis AB, Haugen E, Shafer A, Fu W, Vernot B, Reynolds A, Raubitschek A, Ziegler S, LeProust EM, Akey JM, et al: Exonic transcription factor binding directs codon choice and affects protein evolution. Science 342: 1367-1372, 2013.

20. Waldrop E, Al-Obaide MAI and Vasylyeva TL: GANAB and PKD1 variations in a 12 years old female patient with early onset of autosomal dominant polycystic kidney disease. Front Genet 10: 44, 2019.

21. Khan AH, Lin A and Smith DJ: Discovery and characterization of human exonic transcriptional regulatory elements. PLoS One 7: e46098, 2012.

22. Castellanos M, Mothi N and Muñoz V: Eukaryotic transcription factors can track and control their target genes using DNA antennas. Nat Commun 11: 540, 2020.

23. Fontrodona N, Aubé F, Claude JB, Polvèche H, Lemaire S, Tranchevent LC, Modolo L, Mortreux F, Bourgeois CF and Auboeuf D: Interplay between coding and exonic splicing regulatory sequences. Genome Res 29: 711-722, 2019.

24. Joynt AT, Evans TA, Pellicore MJ, Davis-Marcisak EF, Aksit MA, Eastman AC, Patel SU, Paul KC, Osorio DL, Bowling AD, et al: Evaluation of both exonic and intronic variants for effects on RNA splicing allows for accurate assessment of the effectiveness of precision therapies. PLoS Genet 16: e1009100, 2020.

25. National Center for Biotechnology Information: Genomic variation as it relates to human health (ClinVar); [VCV000429811.3]. Available from: https://www.ncbi.nlm.nih.gov/clinvar/variation/ VCV000429811.3. Accessed October 24, 2021.

26. Roca X, Sachidanandam R and Krainer AR: Intrinsic differences between authentic and cryptic 5' splice sites. Nucleic Acids Res 31: 6321-6333, 2003.

27. Anna A and Monika G: Splicing mutations in human genetic disorders: Examples, detection, and confirmation. J Appl Genet 59: 253-268, 2018.

This work is licensed under a Creative Commons Attribution-NonCommercial-NoDerivatives 4.0 International (CC BY-NC-ND 4.0) License. 